\title{
An Opportunity Networks Routing Algorithm Based On Social Model
}

\author{
Tian zhuo ${ }^{1}$, Libaicheng ${ }^{2}$
}

Information teaching and Management Center, Jilin Agricultural University, Changchun, Jilin, China

xiaomarkshaoqing@163.com,Ibc@163.com

Keywords: opportunistic networks; opportunistic routing; social; relationship degree

\begin{abstract}
In this paper, an opportunity networks routing algorithm, which is suitable a certain social model, is proposed based on social. In this algorithm, the relationship degree between nodes is computed based on the social nature; and the central of nodes is computed based on the level of activity in opportunity networks. An opportunity networks routing algorithm ComOR, which achieves selective forwarding data packets by using the central and relationship degree of nodes,is proposed. Simulation results show that the routing algorithm significantly reduce the consumption of buffer space of equipments and effectively reduce the count of forwarding data packets compared with the present algorithms.In a word,ComOR improves the packets delivery rate and performances stably.
\end{abstract}

\section{Introduction}

Currently, opportunity networks is one of the hottest research fields. Reference give a survey of opportunity networks. One descriptive definition of the opportunity networks: Opportunity networks is a special kind of mobile ad hoc networks, in which the communication range of the mobile node is limited. At the same time, the nodes are sparsely distributed in the deployment area. Moreover, the movement of nodes often led to the network disconnected.

Opportunity networks is an important evolution based on mobile ad hoc networks. In opportunity networks, the nodes achieve communication through move, meet-and-forward between disconnected networks, the traditional mobile ad hoc networks routing algorithms can't run effectively in it.There is some important research about routing algorithms in opportunity networks, such as increasing data arrival rate,minimizing delays in data transmission process, reducing the total resource consumption in the data transfer process, the movement characteristics of network nodes and so on.

Currently, a number of suitable routing algorithms based on opportunity networks have been proposed, such as Epidemic Routing ${ }^{[1]}$, Context-Aware Routing (CAR) ,PROPHET , Prioritized Epidemic (PREP), etc, which are usually referred as opportunity routing algorithms.Epidemic routing and PROPHET routing are two of the classical algorithms. Epidemic routing is the first algorithm applied in opportunity networks. The main design of the algorithm is "storage - carry forward", in which the messages are forwarding by flooding. Routing proliferation may be the best solution when the resources are abundant in the network, because messages can reach the target node within the minimum delay. However, in opportunity networks, the capacity, energy, cache size of nodes, network bandwidth and other resources are often limited, the limited resources often can't satisfy Epidemic routing. PROPHET routing is an improved Epidemic routing, which is a probability estimate routing algorithm. In the algorithm,intermediate nodes selectively forward data packets according to the success rate of data transmission.

\section{Comor Routing Algorithm}

Sociality is an important feature of human society.Human society can be divided into a number of communities.Individuals in the same community tend to maintain closely contact, but rarely contact with the individual of other communities. Sociality mainly represents the regional differences and the relationship between individuals. We can measure the contact between individuals by using relationship degree. There are many kinds of relationship between people, such 
as family, colleagues, friends,classmates, relatives, neighbors, etc. These people contact frequently and closely, the relationship degree between them is high. There is little contact between strangers, therefor, the relationship between strangers is estranged. Some even do not contact with each other, the relationship degree between them is low.

Central is another important feature of human society.Individuals have different movement patterns even they are in the same community, because they paly different roles in the society. Some individuals show more active than others.

The frequency that they contact with other individuals is higher. These people have strong central. The data analysis result in reference ${ }^{[2]}$ shows that only a very small number of nodes in the networks have strong central, most of the nodes have much lower central. This result is consistent with people's intuition. For example, compared with the general public, salesmen or drivers often contact with more people, which makes them stronger central.

The networks with a certain social model shows in Figure1, here we call it social model network, communities are divided according to geographical location. For example, the city with concentration population can be divided into a community, some nodes in the community are more active than others, such as S1, S2, S3, S4, they can not only move in a community but also move between communities. Except the active nodes, other nodes, such as S, D, only move in the community generally. In addition, some nodes in remote areas may not belong to any community, they become isolated nodes.

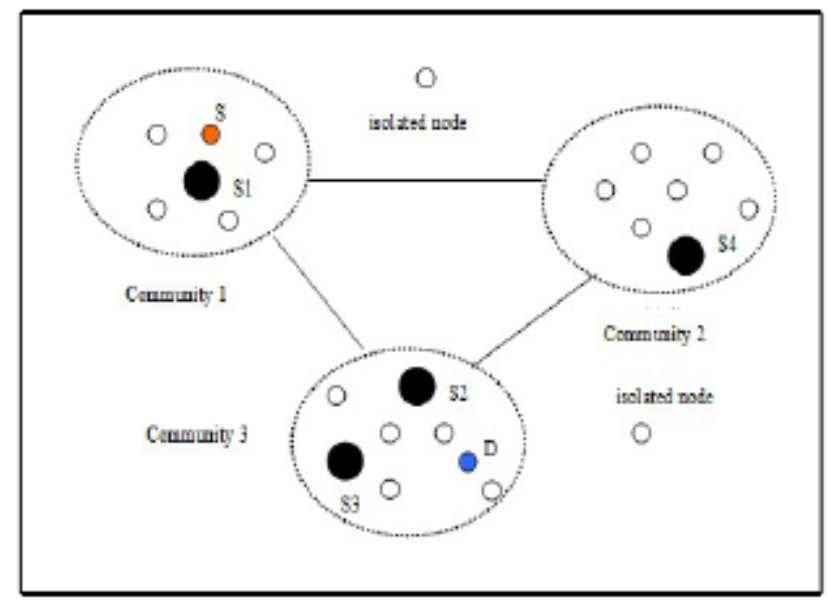

Figure 1. Topology of social model network

In this paper, ComOR is proposed by the characteristics of human society. The algorithm is more suitable for the networks with certain social model, it is more efficient in such networks.

The calculation of relationship degree between two nodes.In the social model networks, the nodes have the characteristics of society, The more intimate relationship between two nodes, the more frequently they contact with each other. We can measure the relationship degree between nodes according to the contact times in a period of time. We define the relationship degree L(i, j) as follows:

$$
L(i, j)=\frac{m_{t(i, j)}}{\sum_{j=1: i \neq j}^{N} m_{t(i, j)}}
$$

It is used to describe the relationship degree between two nodes, the higher value of $L(i, j)$, the higher relationship degree and closer contact between two nodes. In this formula, $\mathrm{N}$ is the count of nodes in the network, $\mathrm{mt}(\mathrm{i}, \mathrm{j})$ is the contact times between node $\mathrm{i}$ and node $\mathrm{j}$ within a recent period of time t. The value of $t$ is setted according to the cyclical movement of nodes in the network. For example, the movement cycle of human is a day or one week. The formula above indicates that the more frequently contact between two nodes the higher value of $L(i, j)$, and $L(i, j) \in[0,1]$.

The calculation of the central of nodes. Some network environment can be considered as composition of many communities, the majority nodes only contact with the other nodes in the same community, only a few nodes contact with the nodes in other communities. In figure 1, S1, S2, 
S3 and S4 can contact other communities that have stronger central, S is the source node, D is the destination node. Suppose S want to send a message to D,they can not directly access, so the central node S1 is the best choice to forward. Freeman ${ }^{[3]}$ proposed several methods to measure the central of nodes. This paper uses the method that the number of nodes met by node $i$ in the past period oftime $t$ divided by the total number of nodes.The formula:

$$
C_{i}=\frac{\sum_{j=1: i \neq j}^{N} s_{i(i, j)}}{N}
$$

It is used to describe the central of a node, the higher value of $\mathrm{Ci}$, the stronger central of the node. $\mathrm{N}$ is the total nodes in network. In the past period of time $t$, if node $\mathrm{i}$ and node $\mathrm{j}$ had a connection, then $\operatorname{St}(i, j)=1$, else $\operatorname{St}(i, j)=0$. The value of $t$ can be setted according to the movement cyclical of nodes in network, here in order to synchronize with $\mathrm{L}(\mathrm{i}, \mathrm{j})$,the movement cyclical is set according to the movement cyclical of $\mathrm{L}(\mathrm{i}, \mathrm{j})$. The formula above indicates that the more active a node the higher value of its $\mathrm{Ci}$, and $\mathrm{Ci} \in[0,1]$.

The calculation of $\mathbf{P i}(\mathbf{j})$.In ComOR algorithm, every node i maintains a transmission probability table(TP_Table), which records the transmission probability from i to every known destination node $\mathrm{j} . \mathrm{Pi}(\mathrm{j})$ is used to represent the successful transmission probability of data packets from node $\mathrm{i}$ to node $\mathrm{j}$. The definition of $\mathrm{Pi}(\mathrm{j})$ is as follows:

$$
\operatorname{Pi}(j)=\mathrm{Ci} \times \mathrm{L}(\mathrm{i}, \mathrm{j})
$$

$\mathrm{Ci}$ is the central of the node $\mathrm{i}, \mathrm{L}(\mathrm{i}, \mathrm{j})$ is the relationship degree between two nodes. After updating $\mathrm{Ci}$, the nodes will update $\mathrm{Pi}(\mathrm{j})$. The update cycle synchronize with the update.

\section{Simulation and Result Analysis}

Experiment verify indicators.In order to verify the performance of ComOR routing algorithms, the following indicators are used to measure the performance:

1) delivery ratio, which present the value of the successful delivery of the messages divided by the total number of the original message.

2) the average delay, which refers to the average delay of all the successful delivery of messages.

3) The average consumption of the node cache, which presents the number of messages carried by each node at the end of the experiment ${ }^{[4]}$, in other words, it presents the consumption of cache.

Experiment settings.On this paper, we use Opportunistic Network Environment (ONE) to do the simulation experiments. The scenario is a $4000 \mathrm{~m} \times 4000 \mathrm{~m}$ area where three communities are distributed. The total number of nodes is 90 . Most of the nodes in each community (75\%) move in their own community, only a few nodes (25\%) move in three communities, the nodes select the shortest path from the source to the destination. The common nodes move in their own community with speeds of $0.5-1.5 \mathrm{~m} / \mathrm{s}$, which equivalent to the speed of human walking. The active nodes move between communities with speeds of $10-25 \mathrm{~m} / \mathrm{s}$, which equivalent to the speed of vehicles and other transport.The communication radius of nodes is $50 \mathrm{~m}$, the cache of a node can accommodate 500 messages at the most. There is sufficient bandwidth between nodes to complete the exchange of each message. The time threshold t of ComOR algorithm to obtain the node central is 100 seconds

Experiment results. The figure 2 shows the performances of ComOR algorithm and other routing algorithms when the routing hops is changing.

From figure 2 (a), the delivery ratio of ComOR is lower than Epidemic when the hops is less than 7. But when the hops is more than 7, the delivery ratio of ComOR is obviously higher than the other two algorithms, and the performance of ComOR is more stable. When the hops increasing, many message copies produced in Epidemic and PROPHET, but this not happened in the ComOR routing.From figure 2 (b), the delay of Epidemic is shorter than both ComOR and PROPHET, In the case of abundant resources,Epidemic algorithm can achieve sending information to the destination node with minimum delay ${ }^{[5]}$. With the increase of hops, the three algorithms are gradually reducing the delay.ComOR can reduce the delay to a very low level, which can be accepted. From figure 2 
(c), the consumption of resources of ComOR are obviously less than both Epidemic and PROPHET. ComOR has effectively controlled the consumption of cache.

From the above results we can know that the performance of ComOR is the best of the three routing algorithms when the routing hops are unlimited.

\section{Conclusions}

In this paper, we discussed the social of the nodes in opportunistic networks and analyzed the necessity for introducing the social to routing algorithm. An pportunistic rooting algorithm based on the social of nodes is proposed.In this algorithm, we calculated the relationship degree $\mathrm{L}(\mathrm{i}, \mathrm{j})$ between nodes by using the social of nodes, at the same time,we also calculated the central $\mathrm{Ci}$ of nodes according to the activity level of nodes in opportunistic network, In-depth probe into the mobility model of node with social and the treatment of isolated nodes in communication, will be the next focus of the study.

\section{References}

[1]C-M Huang,K-c Lan,C-Z Tsai.A survey of opportunistic networks.Proc of 22nd International Conference on Advanced Information Networking and Applications,2008.

[2]Vahdat A,Becker D.Epidemic routing for partially connected ad hoc networks[R].[S.l.]:Duke University,2000.

[3]Musolesi M,Hailes S,et al. Adaptive routing for intermittently connected mobile ad hoc networks [A].Proceedings of WOWMOM'2005[C].Taormina:IEEEComputerSociety Press,2005.813-820.

[4]Lindgren A,Doria A,Schelen O.Probabilistic routing in intermittently connected networks[J].ACM SIGMOBILE Mobile Computing and Communications Review,2003,7(3):19-20.

[5]Ram R,Richard H,et al.Prioritized Epidemic Routing for Opportunistic Networks[C]. MobiOpp. 2007. 